\title{
Pregnancy of unknown viability or location: less is more in the majority of cases
}

\author{
Nick Raine-Fenning, ${ }^{1}$ Shyamaly Sur, ${ }^{2}$ Andrew W Horne, ${ }^{3}$ Helen Wilkinson, ${ }^{4}$ Tom Bourne ${ }^{5}$
}

\author{
'Reader in Reproductive \\ Medicine and Surgery, Division \\ of Obstetrics and Gynaecology, \\ School of Clinical Sciences, \\ University of Nottingham, \\ Nottingham, UK \\ ${ }^{2}$ Clinical Research Fellow in \\ Reproductive Medicine, Division \\ of Obstetrics and Gynaecology, \\ School of Clinical Sciences, \\ University of Nottingham, \\ Nottingham, UK \\ ${ }^{3}$ Senior Lecturer and Consultant \\ Gynaecologist, MRC Centre for \\ Reproductive Health, University \\ of Edinburgh, Edinburgh, UK \\ ${ }^{4}$ Director, The Ectopic Pregnancy \\ Trust, King's College Hospital, \\ London, UK \\ ${ }^{5}$ Visiting Professor and \\ Consultant Gynaecologist, \\ Institute of Reproductive and \\ Developmental Biology, Imperial \\ College, London, UK and the \\ Katholieke University, Leuven, \\ Belgium
}

\section{Correspondence to}

Dr Nick Raine-Fenning, Division of Obstetrics and Gynaecology, School of Clinical Sciences, D Floor, East Block, Queen's Medical Centre, University of Nottingham,

Nottingham NG7 2UH, UK; nick.raine-fenning@nottingham. ac.uk

Received 4 November 2011 Accepted 4 November 2011

\section{Background}

Ultrasound has become an essential part of any early pregnancy assessment. This is particularly true during the first trimester when an ultrasound examination can confirm the number of fetuses, their viability, size and therefore gestational age. Ultrasound was primarily introduced to accurately date a pregnancy and thereby reduce perinatal mortality by decreasing the prevalence of postmaturity and identify multiple pregnancies. This is still relevant today but ultrasound now offers a great deal more information, which can be used to counsel patients and ensure they are appropriately managed throughout their pregnancy.

\section{Classification of early pregnancy outcomes}

One of the most important developments has been the identification of an ectopic pregnancy, an important cause of maternal morbidity and mortality and one that is often subject to substandard care. When an ultrasound examination is performed in early pregnancy there are three eventualities in terms of the location of the gestation sac: the pregnancy can be correctly located within the endometrial cavity (an intrauterine pregnancy), the pregnancy can be ectopically located (an ectopic pregnancy), or the pregnancy cannot be identified [a pregnancy of unknown location (PUL)]. ${ }^{1}$ Intrauterine pregnancies may be viable, non-viable or of uncertain viability. The latter has been a subject of great debate in the media of late and national guidelines have recently been modified to extend the period during which non-viability can be confidently diagnosed to avoid inadvertent termination of a potentially viable pregnancy. ${ }^{2-4}$ These papers highlight the risks that may be associated with developing protocols in early pregnancy on the basis of insufficient data or 'expert opinion' alone. ${ }^{5}$
Ectopic pregnancies can exist outside of the uterus (a tubal or much less commonly an ovarian or abdominal pregnancy) or ectopically within the uterus (interstitial, cervical and Caesarean scar ectopics). Ultrasound can be used to positively identify the location of the ectopic pregnancy, which is paramount as the risks and management options differ considerably involving a variety of conservative, medical and surgical approaches. Over 70\% of tubal ectopic pregnancies can be visualised at the time of an initial examination and over $90 \%$ prior to surgical intervention. ${ }^{6}$ An inappropriately radical surgical approach in an ectopically located uterine pregnancy could result in uncontrollable haemorrhage. ${ }^{7}$ These types of ectopic pregnancy are becoming increasingly common but thanks to the widespread availability and application of ultrasound are also being increasingly recognised and managed appropriately, although more work and possibly a national register are required.

\section{Challenges in early pregnancy diagnosis}

It is the inability to locate a pregnancy that actually poses one of the principal difficulties in today's early pregnancy assessment unit. When a pregnancy cannot be located the same possibilities outlined above still apply in that the pregnancy must be either intrauterine or ectopically located. The pregnancy may be viable or non-viable in either scenario. The challenge is to identify the patient with the ectopic pregnancy at risk of rupture without unduly over-investigating the patient with a viable or nonviable intrauterine pregnancy so as not to exacerbate the level of anxiety to the patient and to reduce unnecessary medical intervention. This is easier said than done. Time will ultimately reveal all eventualities and while, in some cases, the location of the pregnancy is never clarified, 
a conservative approach can be safely adopted in the majority of cases. ${ }^{910}$ The mainstay of management is the serial measurement of serum human chorionic gonadotrophin (hCG) levels, often usefully expressed as the hCG ratio, and in some cases the measurement of serum progesterone. ${ }^{11} 12$ The trend has been towards using these assessments to identify failing pregnancies or viable intrauterine pregnancies that do not require intensive follow-up. A subgroup of patients with a PUL considered to be at a higher risk of having an ectopic pregnancy can be identified and followed up more closely until the location and behaviour of the pregnancy is known. Although still used in some clinics, a single 'threshold' value of hCG is unhelpful for locating a pregnancy as the majority of ectopic pregnancies seen in clinical practice lie below the commonly quoted value of $1000 \mathrm{IU} / \mathrm{l}^{13}$

One common theme both for the management of intrauterine pregnancy of uncertain viability (IPUV) and PUL is that time will reveal the outcome in all cases. In the event of a possible miscarriage, conservative management is a safe and well-researched approach. Intervention for an IPUV, if inappropriate, may lead to inadvertent termination of a wanted pregnancy while doing nothing is unlikely to cause harm. The same may be said of PUL. Given that $10 \%$ to $30 \%$ of PULs are subsequently shown to be an ectopic pregnancy, it is clear that intervention is unnecessary in the majority of cases. Such intervention may take the form of curettage, methotrexate treatment or laparoscopy, all of which have their limitations: Condous et al. have shown that some protocols based on curettage for the management of PUL will be associated with termination of wanted pregnancies, ${ }^{14}$ the inappropriate administration of methotrexate for PUL is currently one of the commonest causes of litigation in the USA, and a negative laparoscopy for ectopic pregnancy is considered a risk issue and an indication of the standard of care in some units.

\section{Recent CMACE recommendations}

It is against this background that the commentary in the most recent Centre for Maternal and Child Enquiries (CMACE) report ${ }^{15}$ was written which suggested that “... the term 'pregnancy of unknown location' based on early pregnancy ultrasound examination should be abandoned" and that "An early pregnancy ultrasound which fails to identify an intrauterine sac should stimulate active exclusion of tubal pregnancy, and even in the presence of a small uterine sac, ectopic pregnancy cannot be excluded". This statement is at odds with both the current scientific evidence and clinical experience and, in our opinion, would signify a retrograde step in the management of early pregnancy problems as it would lead to unnecessary intervention in many of cases. The consequences of such intervention have been shown starkly by the recent Irish National Miscarriage Misdiagnosis Review ${ }^{16}$ commissioned by the Health Service Executive in April 2011. It would be unfortunate to find similar reports being necessary for the management of PUL. However we feel that the use of the phrase "stimulate active exclusion of" may create pressure on clinicians to force a diagnosis to be made at the time of the initial examination. In practice most women characterised as having a PUL should be reassured but clearly told that there is a possibility of an ectopic pregnancy and given written information about what to do should she develop symptoms of concern.

The CMACE report has been an important development and is embedded in the culture of our specialty. Learning from mistakes made is clearly essential but the majority of medical errors are complex and usually relate to a breakdown of 'the system'. The writers of the CMACE report are in the privileged position of having access to the records of the patients involved in the report. However if wide-scale changes in practice are to be recommended in the report, then these should only be implemented after taking into account the evidence base and not on the basis of single case reports. Such change should also be considered after consultation with clinicians and other health care practitioners working in the field of early pregnancy care. The cases described in the report contain numerous errors relating to the quality of ultrasonography, clinical decision-making, supervision and surgery. We can call the clinical situation of a PUL anything we like, but correct management relies on basic competency in ultrasound, the ability to follow a protocol, knowing when to ask for help, and the appropriate delegation of responsibility. Nomenclature seems the least of our worries.

Perhaps predictably, the CMACE report attracted critical correspondence to the British Journal of Obstetrics and Gynaecology (BJOG) in relation to the chapter relating to deaths in early pregnancy ${ }^{17-19}$ and raised questions about the use of BJOG for the publication of articles that are not subject to the same peer review process of scientific papers submitted to the journal itself. The response to the correspondence did not offer any justification for the assertion that the term PUL should be abandoned and did not address some of the important questions that were raised. We still do not know what is meant in the context of miscarriage: "Persistent bleeding, as was found in a number of women, needs to be recognised as an indication for prompt surgical evacuation of retained products of conception". Does persistent mean that it is heavy, prolonged or both? In isolation it could mean performing surgery in nearly all women with a miscarriage as they nearly all bleed for a period of time. Similarly, the phrase "active exclusion" is impossible to interpret. In the authors' response we were also assured that no Caesarean section scar ectopic pregnancies were associated with maternal deaths. However, elsewhere in the report we are informed that "In three deaths, 
all occurring at 16-18 weeks of gestation, massive and ultimately uncontrollable haemorrhage was associated with placental localisation at the site of a previous lower segment Caesarean section scar". Furthermore the issue of "morbid adherence of the placenta to the scar" is prominently located as an essential learning point of the chapter. Given this information it would seem likely that the pregnancy was located in the Caesarean section scar from the outset and so by definition was ectopic from the outset. What did the detailed review of the scans associated with the case show and what are the learning points? Perhaps the major problem in these cases was the fact that implantation in the scar was not identified earlier so that intervention could have been relatively safe. Unfortunately we will never know the answer to these questions.

Perhaps what we are seeing with CMACE is a clash of cultures. A parallel can be seen in oncology. At one time the emphasis seemed to be on relatively aggressive surgery. Over time the approach has become far more nuanced, with fertility sparing being a priority in younger women and surgery taking into account later function as well as tumour clearance. In early pregnancy care the standard management for both miscarriage and a PUL was surgery. This has changed. We now rarely need to operate on women with an incomplete miscarriage, and a false-negative laparoscopy for ectopic pregnancy is always disappointing. The emphasis is on early diagnosis of non-tubal ectopic pregnancy and less aggressive intervention. This is why the CMACE report is disappointing as it reflects the type of early pregnancy care seen of over a decade ago. The concerns raised about the report are important as there is a risk that CMACE will lose credibility if these issues are not addressed. If the confidential enquiry is to make wider recommendations about patient care and protocols at a national level then these must be informed by the evidence base, the information from the cases themselves and expert opinion. One out of three will not do.

\section{Concluding remarks}

The term 'pregnancy of unknown location' has become an established part of clinical practice both within Europe and the USA. ${ }^{1}$ It reminds health care practitioners not only of the possibility of ectopic pregnancy but also that the pregnancy may be normally located but too small to see or non-viable such that it has failed to develop in accordance with the gestational age based on the last menstrual period. It is important to remember that the location of a pregnancy cannot be determined in between $5 \%$ and $31 \%$ of women attending hospital with early pregnancy problems ${ }^{20}$ but only $6-9 \%$ of these women will eventually be diagnosed with an ectopic pregnancy. ${ }^{21}$ Not only is 'pregnancy of unknown location' an important term for these reasons, it is also the only term that truly describes the clinical situation. It is, therefore, a term we endorse, and one that we hope will continue to ensure that our patients are appropriately managed in an era where a conservative, safe approach is possible through the judicious use of ultrasound and serum biochemistry.

\section{Competing interests None.}

Provenance and peer review Commissioned; internally peer reviewed.

\section{References}

1 Barnhart K, van Mello NM, Bourne T, et al. Pregnancy of unknown location: a consensus statement of nomenclature, definitions, and outcome. Fertil Steril 2011;95:857-866.

2 Abdallah Y, Daemen A, Guha S, et al. Gestational sac and embryonic growth are not useful as criteria to define miscarriage: a multicenter observational study. Ultrasound Obstet Gynecol 2011;38:503-509.

3 Abdallah Y, Daemen A, Kirk E, et al. Limitations of current definitions of miscarriage using mean gestational sac diameter and crown-rump length measurements: a multicenter observational study. Ultrasound Obstet Gynecol 2011;38: 497-502.

4 Pexsters A, Luts J, Van Schoubroeck D, et al. Clinical implications of intra- and interobserver reproducibility of transvaginal sonographic measurement of gestational sac and crown-rump length at 6-9 weeks' gestation. Ultrasound Obstet Gynecol 2011;38:510-515.

5 Wise J. Current ultrasonographic criteria for miscarriage could lead to misdiagnosis, say researchers. BMJ 2011;343:d6665.

6 Kirk E, Condous G, Bourne T. Pregnancies of unknown location. Best Pract Res Clin Obstet Gynaecol 2009;23: 493-499.

7 Takeda A, Manabe S, Mitsui T, et al. Management of patients with ectopic pregnancy with massive hemoperitoneum by laparoscopic surgery with intraoperative autologous blood transfusion. J Minim Invasive Gynecol 2006;13:43-48.

8 Savvidou MD, Setchell TE, Sieunarine K, et al. Conservative surgical management of ruptured interstitial pregnancy. Acta Obstet Gynecol Scand 2006;85:629-631.

9 Banerjee S, Aslam N, Woelfer B, et al. Expectant management of early pregnancies of unknown location: a prospective evaluation of methods to predict spontaneous resolution of pregnancy. BJOG 2001;108:158-163.

10 Banerjee S, Aslam N, Zosmer N, et al. The expectant management of women with early pregnancy of unknown location. Ultrasound Obstet Gynecol 1999;14:231-236.

11 Bignardi T, Condous G, Alhamdan D, et al. The hCG ratio can predict the ultimate viability of the intrauterine pregnancies of uncertain viability in the pregnancy of unknown location population. Hum Reprod 2008;23:1964-1967.

12 Bignardi T, Condous G, Kirk E, et al. Viability of intrauterine pregnancy in women with pregnancy of unknown location: prediction using human chorionic gonadotropin ratio vs. progesterone. Ultrasound Obstet Gynecol 2010;35:656-661.

13 Condous G, Kirk E, Lu C, et al. Diagnostic accuracy of varying discriminatory zones for the prediction of ectopic pregnancy in women with a pregnancy of unknown location. Ultrasound Obstet Gynecol 2005;26:770-775.

14 Condous G, Kirk E, Lu C, et al. There is no role for uterine curettage in the contemporary diagnostic workup of women with a pregnancy of unknown location. Hum Reprod 2006;21:2706-2710. 
15 Centre for Maternal and Child Enquiries (CMACE). Saving Mothers' Lives: Reviewing Maternal Deaths to Make Motherhood Safer: 2006-2008. The Eighth Report of the Confidential Enquiries Into Maternal Deaths in the United Kingdom. BJOG 2011;118(Suppl. 1):1-203.

16 Health Service Executive. National Miscarriage Misdiagnosis Review. 2011. http://www.hse.ie/eng/services/Publications/ services/Hospitals/miscarriagereview.html [accessed 3 November 2011].

17 Condous G. The term 'Pregnancy of unknown location' is staying put. BJOG 2011;118:1402; discussion 1403-1402; discussion 1404.
18 Wilkinson H. Saving Mothers' Lives. Reviewing maternal deaths to make motherhood safer: 2006-2008. BJOG 2011;

118:1402-1403; discussion 1403-1404.

19 Ben-Nagi J, Jurkovic D. Deaths in early pregnancy. The Eighth Report of the Confidential Enquiries Into Maternal Deaths in the United Kingdom. BJOG 2011;118:1401-1402; discussion 1403.

20 Condous G, Timmerman D, Goldstein S, et al. Pregnancies of unknown location: consensus statement. Ultrasound Obstet Gynecol 2006;28:121-122.

21 Bottomley C, Van Belle V, Mukri F, et al. The optimal timing of an ultrasound scan to assess the location and viability of an early pregnancy. Hum Reprod 2009;24:1811-1817. 